Revue d'histoire de l'enfance « irrégulière »

Le Temps de l'histoire

1 | 1998

La protection de l'enfance : regards

\title{
Quelques réflexions sur le travail du groupe "Histoire de l'IPES de Belle Ile-en-Mer"
}

Michel Illiaquer

\section{(2) OpenEdition \\ Journals}

Édition électronique

URL : http://journals.openedition.org/rhei/22

DOI : $10.4000 /$ rhei.22

ISBN : 978-2-7535-1638-0

ISSN : $1777-540 \mathrm{X}$

Éditeur

Presses universitaires de Rennes

Édition imprimée

Date de publication : 15 novembre 1998

Pagination : 176-180

ISSN : 1287-2431

Référence électronique

Michel Illiaquer, "Quelques réflexions sur le travail du groupe "Histoire de I'IPES de Belle lle-en-Mer" », Revue d'histoire de l'enfance « irrégulière » [En ligne], 1 | 1998, mis en ligne le 16 mai 2007, consulté le 03 décembre 2020. URL : http://journals.openedition.org/rhei/22 ; DOI : https://doi.org/10.4000/rhei.22 


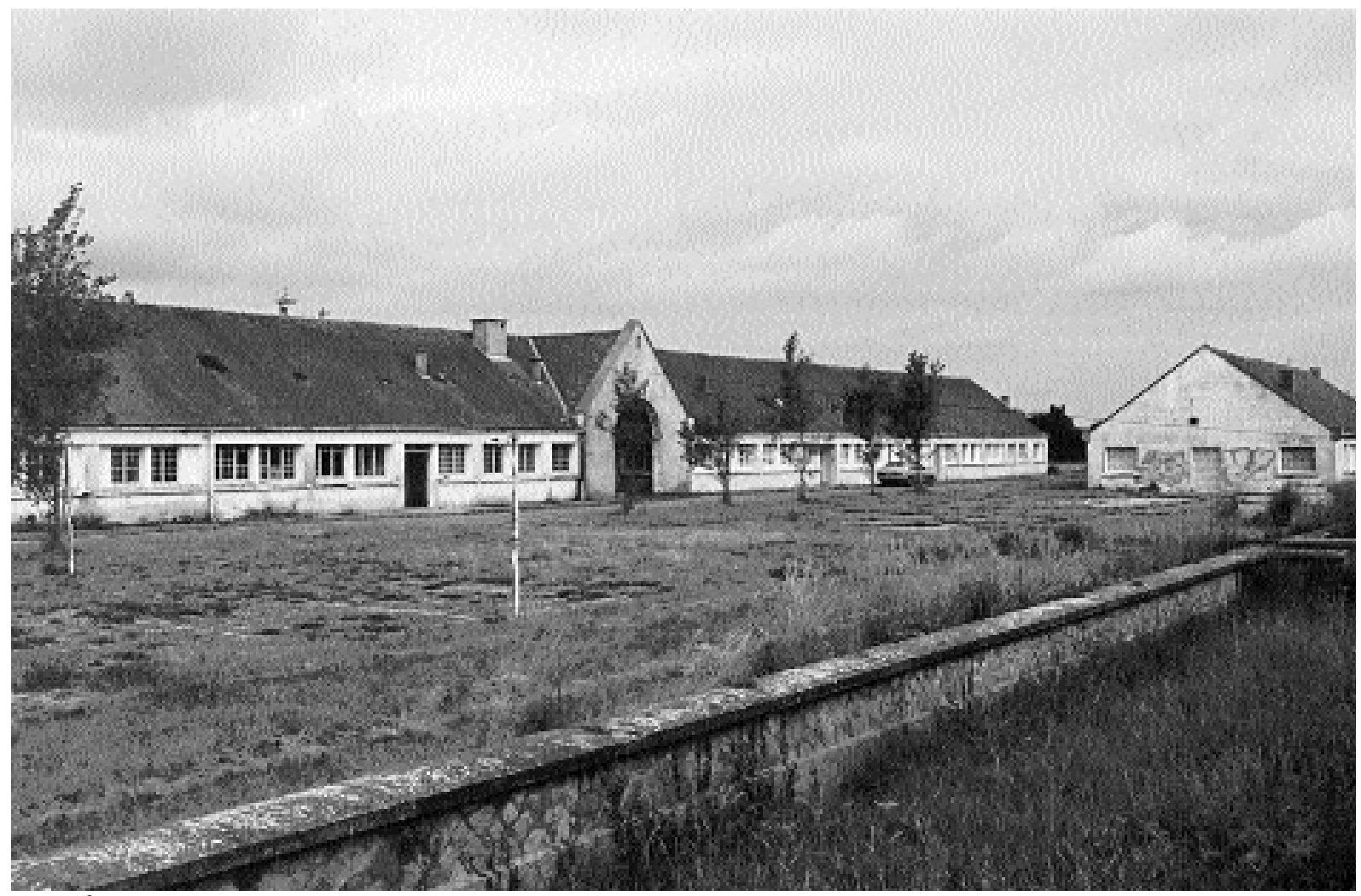

Belle-Île-en-Mer

Haute-Boulogne

internat et cuisine, réfectoire

[Michel Basdevant, 1997] 


\section{Quelques réflexions sur}

\section{le travail du groupe "Histoire de I'IPES de Belle-Île-en-Mer"}

\author{
"L'bistoire est la reconstruction toujours problématique et incomplète \\ de ce qui n'est plus. La mémoire installe le souvenir du sacré, l'bistoire l'en \\ débusque. " ${ }^{(2)}$ \\ "L'bistoire objectivée, instituée, ne devient action historique, c'est-à-dire \\ histoire agie et agissante que si elle est prise en charge par des agents que leur \\ histoire prédispose à l'assurer, et qui, du fait de leurs investissements \\ antérieurs, sont inclinés à s'intéresser à son fonctionnement et sont dotés des \\ aptitudes nécessaires pour la faire fonctionner. " ${ }^{(3)}$
}

1er avril 1996. Dernière réunion à Rennes. C’est fini. Nous avons sous les yeux le résultat de plus de deux années de travail. Cela fait une drôle d'impression : satisfaction de voir le travail achevé, mais aussi sentiment que quelque chose nous échappe.

\section{"VOGUE LA GALÈRE"}

Et pourtant quel chemin parcouru!

Au départ une idée : pourquoi ne pas faire un travail sur l'histoire de l'institution de Belle-Île-en-Mer? De 1880, création de l'établissement de l'Administration pénitentiaire, à l'Éducation surveillée en 1945, jusqu'à la fermeture en 1977. Vaste programme!

Le Centre régional de formation Bretagne met en place, dès 1994, une session de formation.

Les personnels formant ce groupe avaient des motivations diverses. Un peu de nostalgie sans doute : certains avaient travaillé à Belle-île plus ou moins longtemps. D'autres n'étaient pas Bellilois. Un intérêt aussi pour l'histoire en elle-même : «connaître son passé institutionnel per-

\section{Michel \\ Illiaquer $^{(1)}$}

(1) Éducateur à la Protection judiciaire de la jeunesse.

(2) Pierre Nora. Les lieux de mémoire, tome I, Paris, Gallimard, 1984, p. XIX.

(3) Pierre Bourdieu, "Le mort saisit le vif", Actes de la recherche en sciences sociales, 31,1980 , p. 6.

(4) Institution publique d'éducation surveillée. 
met de gérer le présent et de se projeter dans l'avenir ". De fait, plusieurs groupes se sont constitués sur l'histoire de l'institution. Des travaux sont déjà réalisés ou en cours. On peut citer, entre autres, les recherches sur Mettray, Bourges, Savigny...

Je me souviens de cette première réunion. On avait l'idée, mais comment allait-on faire? Le champ d'action était vaste : de 1880 à 1977, quasiment un siècle! Comment procéder? Faire un travail complet, de fond, aurait demandé du temps et des moyens que nous n'avions pas.

Notre premier travail a été de définir un cadre, de se fixer un objectif : s'en tenir à la période 1945-1977.

Éviter aussi de tomber dans l'anecdotique et de produire un recueil de petites histoires qui, même si elles font partie du quotidien, n'ont d'originalité que d'avoir été vécues. Mais, là ou ailleurs, quelle différence? Il $\mathrm{y}$ a toujours des anecdotes à raconter, qui ne mènent pas très loin...

La réflexion a pris forme. Que devait être le produit fini : un écrit?, un film? une vidéo? une cassette audio?

Avantage pour notre institution encore jeune, la période retenue, 1945 à la fermeture, permettait d'avoir une mémoire encore vive. Nous pouvons effectivement récolter les témoignages de personnels ayant travaillé à Belle-Île depuis 1945 et aussi d'élèves ayant été placés à l'IPES. ${ }^{(4)}$

Un courrier est paru dans la presse régionale (Ouest-France, le Télégramme de Brest) et nationale. Plusieurs réponses nous sont parvenues d'anciens élèves contents de témoigner de ce qu'ils ont connu, vécu à Belle-Île.

Encore fallait-il une méthode. L'historien Thierry Fillaut, qui a dirigé le groupe, avait l'avantage de ne pas connaître l'Éducation surveillée, donc d'avoir un "œil neuf”. Ce qui nous a évité certaines dérives.

\section{DES PISTES DE TRAVAIL}

- Recherches dans les archives de l'institution à Vaucresson, aux archives départementales du Morbihan. Les rapports annuels, les différents courriers entre l'IPES et l'administration. Tout un travail de recherche, de lecture et de sélection en fonction de l'intérêt des différents écrits. 
- Rencontre des anciens personnels et élèves ayant répondu à l'article paru dans la presse. La règle du jeu était la suivante : rencontrer et enregistrer les témoignages des acteurs. Ensuite, transposer par écrit les entretiens. À partir de ce long travail, il a fallu constituer des fiches par thème. Par exemple : la vie dans les groupes, les récompenses, les punitions, les formations, etc...

Un plan de travail final a ainsi pris forme. Le plan adopté a évolué au fur et à mesure de l'avancement de la recherche. L'intérêt des entretiens était de pouvoir faire des recoupements et donc d'approcher une "vérité historique", tout en sachant qu'au vu de l'étendue du sujet nous ne pouvions faire qu'un survol de la période. Le document final pourrait toujours être amélioré - peut être plus tard - en creusant certains aspects, par exemple en approfondissant certaines "histoires de vie" des jeunes placés à l'IPES.

Il faut préciser, là, le rôle essentiel de Thierry Fillaut qui a su diriger le groupe. Ce groupe a eu deux points forts :

- l'intérêt historique en lui-même : l'histoire et l'évolution de l'institution, à Belle-Île, mais tout aussi bien ailleurs.

- l'aspect formation qui consiste dans le choix de la méthode. Ce qui a été fait peut servir pour d'autres travaux. A quoi s'ajoute la satisfaction d'avoir mené à terme ce travail et abouti à un produit fini.

En terminant, on peut se demander pourquoi une institution si jeune ressent le besoin de se pencher sur son histoire.

Tant les anciens personnels que les anciens élèves, c'est à souligner, ont témoigné "spontanément". Les anciens personnels sans entrer dans l'anecdote, mais avec un souci d'analyse, même si l'affectif apparaissait de temps en temps. Des personnels qui vivaient pour leur travail, quelquefois au détriment de la vie de famille, mais qui en gardaient généralement un bon souvenir. Des anciens élèves qui, pour certains, ont trouvé à Belle-Île un "foyer" qu'ils n'avaient pas ou plus dans leur famille. Cela peut paraitre paradoxal.

Un seul exemple.

Un jeune en section Marine. Deux ans à Belle-Île après d'autres placements dès son plus jeune âge. Lorsqu'il est «libéré», il loue une chambre 
pendant huit jours chez un pêcheur de l'île. "J'ai pris des vacances ", nous dit-il. Il savait qu'en retournant chez sa mère, il retrouverait la même situation, les mêmes problèmes. Ce qui fut le cas.

Les exemples pourraient se multiplier : on retrouve ainsi le "caïd" pour qui le passage à Belle-Île n'a rien changé et qui aura une vie tumultueuse; le "filou" qui aura bien compris le système et qui saura "gérer" le temps de son placement; enfin, le jeune qui aura assimilé "ferme ta gueule, travaille et ne te fais pas repérer ", bosseur, mais aussi quelque part "souffre-douleur".

Nous retrouvons ainsi ce qui fait partie de notre quotidien de travailleur social.

Cette démarche, sur plus de deux années, nous a permis de voir à la fois l'évolution de l'institution, notamment dans le changement des modes de prises en charge des mineurs que nous recevons, et l'importance de la mémoire "collective", tant professionnelle que personnelle, que nous cultivons et qui est partie prenante de notre histoire et de notre identité.

"Il n'y a pas de projet sans mémoire. " 